\title{
Un objectif pour la SFP: doubler le nombre de ses membres
}

Depuis quelques années, de nombreuses actions ont été entreprises à la Société Française de Physique : renouvellement de sa revue Reflets de la Physique; amélioration du site web; mise en place d'une bourse de l'emploi ; création de nouveaux prix de thèse ; actions internationales au Maghreb ; rapprochement avec d'autres sociétés savantes, françaises et européennes, etc. Par ailleurs, au niveau politique national, notre société fait entendre sa voix dans de nombreux domaines, notamment face aux réformes en cours dans I'enseignement et la recherche, par I'envoi d'articles à la presse, en prenant la défense du CNRS, en se préoccupant des débuts de carrière, et en étant reçue par les politiques.

Toutes ces actions correspondent au rôle de "lobbying » attendu d'une société savante et vont continuer à être développées dans le futur. Elles montrent que notre société a entamé une opération de retoilettage en profondeur de ses structures et objectifs. Cependant, en dépit de cette rénovation et de ces actions positives, I'augmentation du nombre des adhérents est faible et ne reflète pas cette évolution. Des améliorations vont être encore apportées : un fonctionnement plus transparent vis-à-vis de l'extérieur, des relais plus nombreux avec les différentes sections de province, des moyens financiers plus adéquats en rapport avec les objectifs... Reste que la comparaison avec les Sociétés de Physique allemande (la DPG) ou britannique (l'loP), beaucoup plus professionnelles et dix fois plus importantes par leur nombre d'adhérents - alors que les communautés scientifiques sont comparables -, donne à réfléchir : après des études de physique en France, les ingénieurs ne se sentent pas forcément d'appartenance à une communauté de physiciens et se reconnaissent plus dans des associations professionnelles que dans la SFP, plus généraliste. Notre société doit s'ouvrir davantage au monde industriel, en attirant des ingénieurs dans son Conseil d'Administration, en leur ouvrant les pages de Reflets et en impliquant ses sections locales.

Force est de constater que le développement de la SFP ne peut se faire que par un recrutement massif chez les jeunes. On constate en effet qu'il y a, en
France comme ailleurs, un attachement profond des jeunes chercheurs pour leur métier. Beaucoup s'impliquent dans la diffusion de la culture scientifique, comme le montrent les activités des membres du Conseil d'Administration, presque tous âgés de moins de 40 ans. La vitalité de la commission "Jeunes de la Physique » en est un autre témoignage. La campagne de promotion de 2006-2007, à $10 €$ pour une première adhésion, a porté quelques fruits, mais est restée trop confidentielle et pas suffisamment assortie d'un suivi personnalisé. Elle va être relancée, avec des objectifs nettement plus ambitieux et plus fortement affichés.

La SFP a sollicité le concours du CNRS et du CEA pour appuyer sa campagne en faisant apparaître des activités conjointes, par exemple dans le domaine de l'information scientifique et de la communication. Les directeurs de laboratoires vont être sollicités pour faciliter les adhésions de tous leurs jeunes. Une présentation de la SFP dans les écoles d'ingénieurs est au programme, ainsi que des contacts avec les étudiants de master dans les universités. Nous allons nous inspirer des bonnes pratiques qui réussissent dans d'autres pays. Ainsi, en Italie, I'adhésion gratuite avec envoi du journal est offerte pour un an à tous les étudiants de licence. En Allemagne, tous les doctorants s'inscrivent à la DPG pour aller gratuitement aux grands congrès de cette société, où ils trouvent une tribune et des offres d'emploi. En Grande-Bretagne, I'loP a multiplié son nombre de membres par 3 en 10 ans, en fidélisant une bonne fraction des étudiants undergraduate, après leur avoir offert une adhésion gratuite présentée comme l'appartenance à un « club» prestigieux.

La bourse aux idées est ouverte. En faisant mieux connaître nos actions, en sortant du milieu universitaire, en montrant aux jeunes physiciens les points positifs qu'ils retireront de leur adhésion à la SFP, nous atteindrons notre objectif premier de croissance : doubler ou tripler le nombre de nos adhérents, de façon durable et irréversible.

Michèle Leduc et Martial Ducloy 